\title{
Optical calibration of the ELT adaptive mirror M4: strategy for optical measurement error estimation
}

\author{
Marco Xompero ${ }^{1}$, Runa Briguglio ${ }^{1}$, Giorgio Pariani ${ }^{2}$, Matteo Tintori ${ }^{3 \text {, }}$ Armando Riccardi ${ }^{1}$, Daniele \\ Gallieni ${ }^{2}$, Roberto Biasi ${ }^{4}$, Elise Vernet ${ }^{5}$, Marc Cayrel $^{5}$ \\ ${ }^{1}$ INAF - Osservatorio Astrofisico di Arcetri, largo E. Fermi 5, 50125 Firenze Italy \\ ${ }^{2}$ INAF - Osservatorio Astronomico di Brera, via E. Bianchi 46, 23807 Merate, Italy \\ ${ }^{3}$ A.D.S International, via Roma 87, 23868 Valmadrera, Italy \\ ${ }^{4}$ Microgate, via Stradivari 4, 39100 Bolzano, Italy \\ ${ }^{5}$ ESO Headquarter, Karl Schwarzschild strasse 2, D-85748 Garching, Germany
}

\begin{abstract}
For the optical characterization of M4 Unit (M4U) is mandatory to set up an opto-mechanical solution that can guarantee excellent measurement quality over the whole $2.4 \mathrm{~m}$ clear aperture.

The final design of the test structure foresees the use of a dynamic interferometer with 1200x1200 pixel resolution that generates a test beam collimated by a $1.5 \mathrm{~m}$ diameter parabolic mirror to image a single M4U segment. The test tower provides the capability to illuminate each single segment plus the center of the entire unit. A reference flat of $600 \mathrm{~m}$ can be inserted to decouple the M4U from the alignment aberrations.

In the paper we will present the strategy for the budgeting of the optical measurements in the Optical Test Tower (OTT). Following a bottom up approach, we identified a set of possible noise sources, then we computed their contribution to the optical measurements, reporting in the end the global effect on the calibration. The M4U project is led by the italian consortium AdOptica under an ESO contract.
\end{abstract}

\section{INTRODUCTION}

The M4U is the adaptive mirror mounted in the ELT optical train ([1]). It will correct mechanical vibrations and the atmospheric seeing. Since it is potentially servicing all the instruments, it is crucial not only to have strong performances during the observations, but also an extreme quality in the calibrations of the system and in their long-term stability. The optical characterization and test will become therefore a fundamental part of the M4 project as it will be the demonstration of the M4 capabilities and it will state the readiness of the system against the ELT requirements.

\section{THE OPTICAL TEST TOWER}

The M4U will be mounted on an Optical Test Tower (OTT). The test tower will provide two measurement configurations which are named LAI (Large Aperture Interferometry) and SAI (Small Aperture Interferometry). The first allows the measurement of a $1.5 \mathrm{~m}$ circle projected on the M4U shell through a large parabolic mirror, the second instead allows the direct measurement of the M4U surface with a $100 \mathrm{~mm}$ beam. The LAI test tower concept and design are briefly illustrated in Figure 1 and Figure 2. ([2]) 


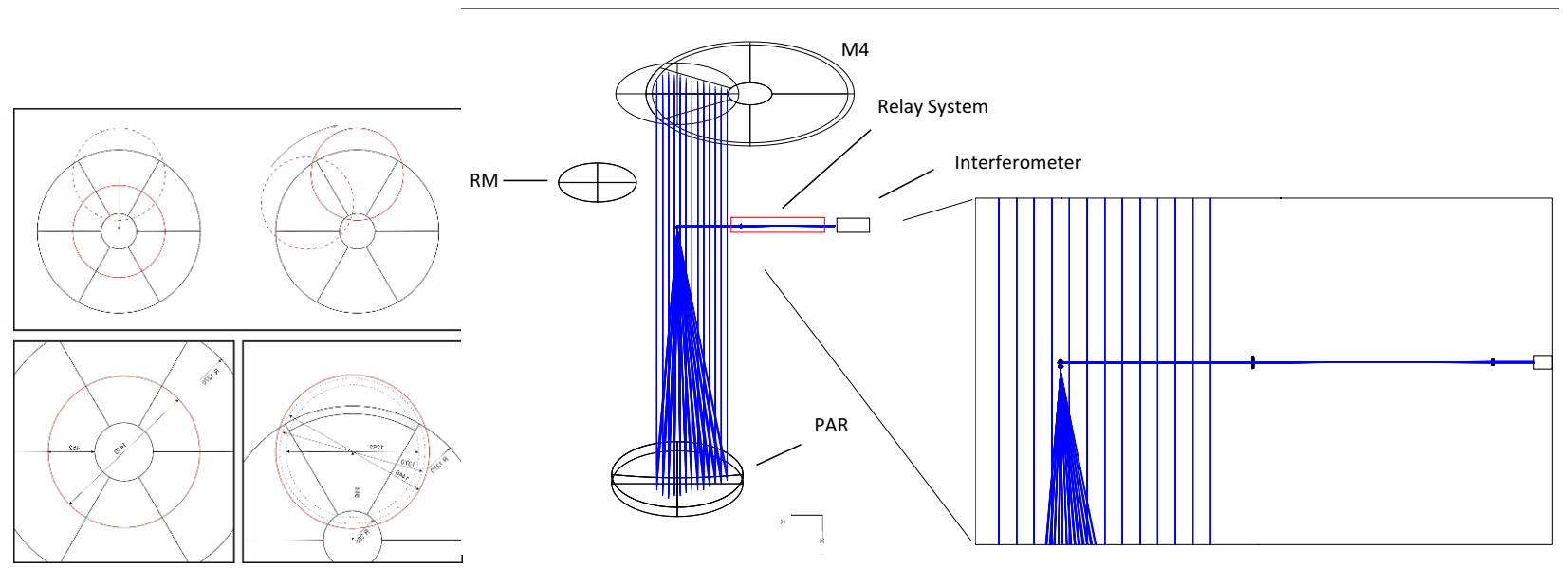

Figure 1: Large Aperture Interferometry configuration optical design
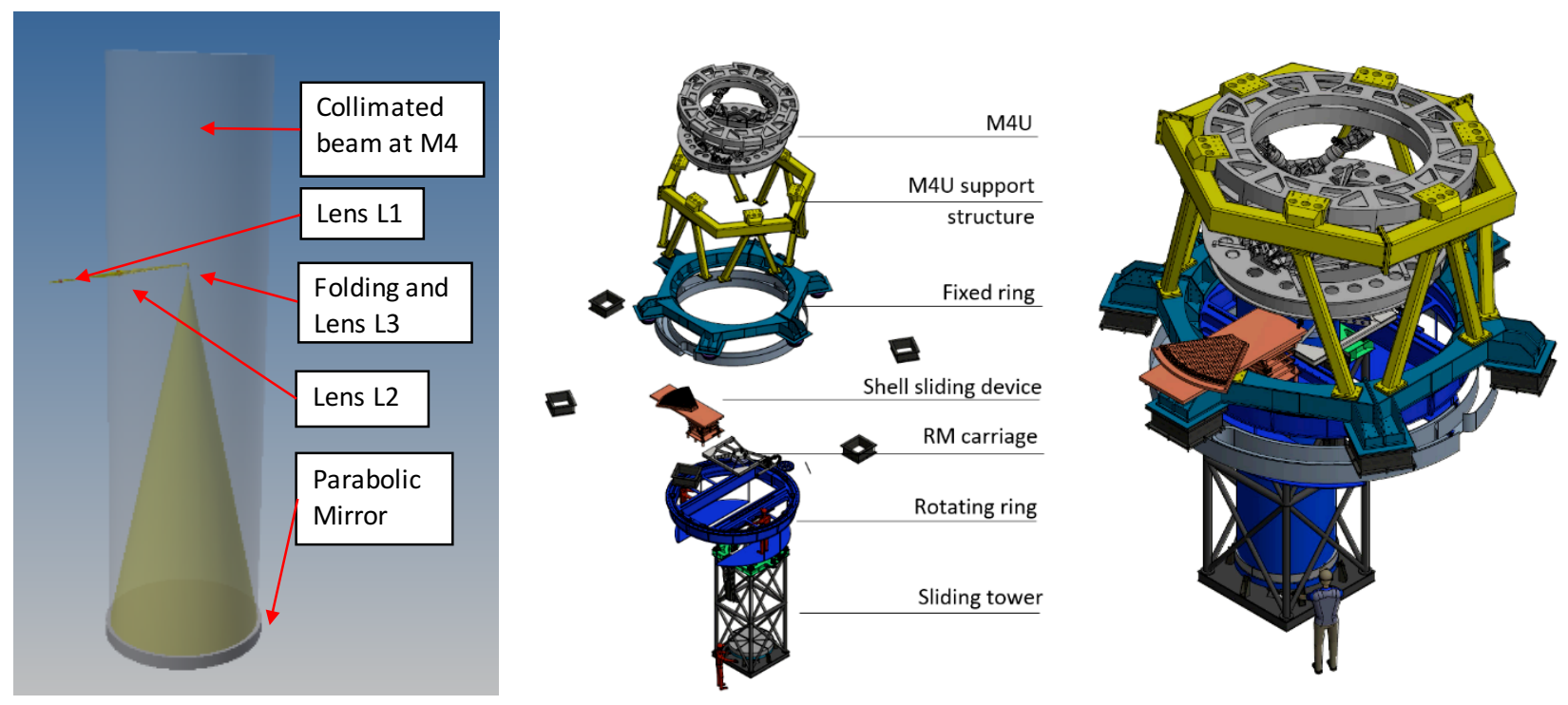

Figure 2: Optical test tower (OTT) mechanical description and design

A rotating ring is sliding the optical tower with the parabolic mirror around the M4U. This degree of freedom allows the interferometric measurement of a whole unit segment plus part of the neighbor segments. The interferometer chosen is the a Twyman-Green interferometer with $840 \times 840$ true meta-pixels on the detector, which allows a resolution of about $1.8 \mathrm{~mm}$ on M4 unit and an acquisition frame rate of $25 \mathrm{~Hz}$.

The M4U is measured in double pass. A $600 \mathrm{~mm}$ bulk reference flat may be inserted for calibration purposes in front of M4U.

In the SAI design the interferometer is installed on the side of the sliding tower with a set of optics set allowing the collimated beam expansion of $100 \mathrm{~mm}$.

In this way is possible to sample the whole surface of M4U with high resolution and without the parabolic mirror effect on the measurements. With using the same interferometer chosen for the LAI configuration, the resulting resolution is $0.150 \mathrm{~mm}$ per pixel.

At the end, an absolute piston sensor (SPL) ([2]) provides an estimation of the true piston value of each segment, despite the interferometer intrinsic phase ambiguity (Figure 3). 


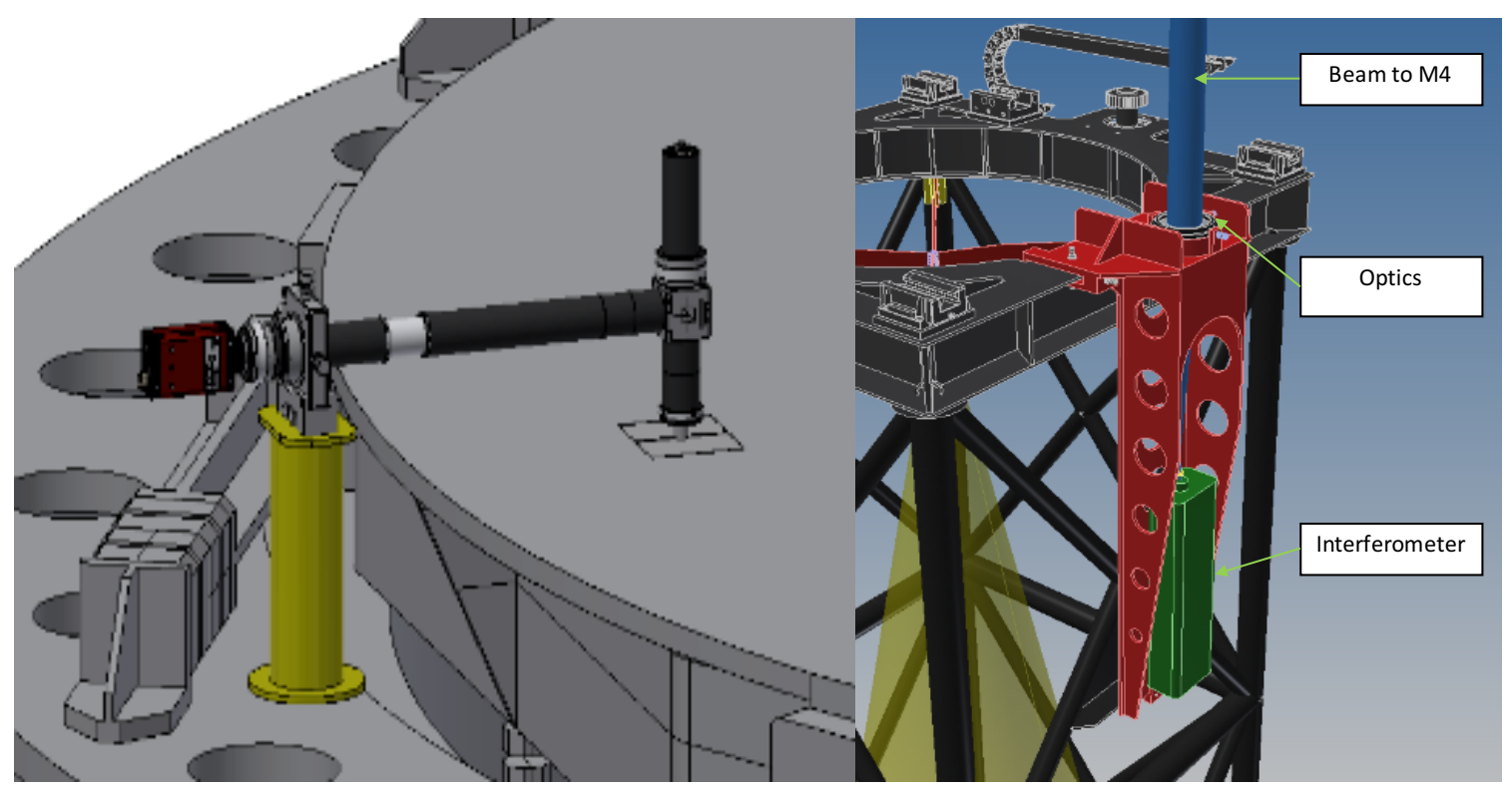

Figure 3: Details of SPL (left) and SAI (right) mechanical implementation.

Each one of the six SPL sensor can be inserted in front of the M4U surface, across the segments. It illuminates with a collimated beam the shell gap and focalize the reflected light onto a detector, recording the diffraction pattern. The analysis of the spot allows the determination of the local piston between the two shells.

\section{MEASUREMENTS STRATEGY}

Before to proceed with the discussion of the analysis strategy against the REQ (REQuirements), is useful to analyze the main measurement strategy that can be applied to the optical test tower.

All the measurements can be essentially grouped in two sets: absolute and differential.

\subsection{Absolute measurements (ABS)}

Absolute measurements allow to capture the actual surface map of the M4U or of the reference flat of the OTT and consists of single or time averaged interferometer realizations. Considering random spectrum noise, the noise effect can be reduced by averaging the measurements and it will decrease according the square root of the number of measurements. On the other side, the absolute measurements are affected by accuracy errors even in terms of systematic noise that cannot be removed so it has to be kept low by design.

Within the OTT we identified the following noise tree:

- $\quad$ Systematic errors

○ optics manufacturing:

- polishing residual

- optics shape thermal drift

- manufacturing reference offset

○ optical alignment residuals

- measurement noise:

$\circ \quad$ interferometer $\mathrm{CCD}$ noise

- air convection 
For each one of them, we can identify the general analysis methods that can be applied to evaluate absolute measurements errors provided by them:

- $\quad$ Systematic errors

optics manufacturing:

- polishing residual: use the reference values from datasheet and literature or perform direct measurement of samples whose manufacturing is comparable with OTT optics;

- optics shape thermal drift: perform a finite element modeling and simulations to evaluate the expected optical aberrations due to the thermal flexures;

- manufacturing reference offset: such error is an unavoidable accuracy offset (without any further corrective action) and is budgeted as a specification for the manufacturer;

○ optical alignment residuals: will be evaluated from the OTT opto-mechanical model after defining an alignment procedure;

- measurement noise:

- air convection: it can be evaluated with a statistical analysis of datasets representing air convection noise taken in environment similar to the OTT.

○ interferometer CCD noise: the analysis can be based on laboratory measurement; in particular, it can be merged together with air convection.

\subsection{Differential measurement errors}

Differential sampling is requested to perform a set of optical calibrations (e.g. actuators influence functions (IF), mirror modes IF, capacitive sensors (capsens) calibration) where an actuator command is applied and captured with the pushpull technique. The same applies for the verification of a REQ which is associated to the optical measurement of an actuator command. The dataset composed by the $n$ frames is analyzed according to a differential algorithm.

While differential samplings are poorly sensitive to offsets and slow drift, they are affected by measurement noise, namely interferometer CCD noise, air convection and turbulence, vibrations, high speed drifts. The measurement noise may be directly evaluated by collecting a test dataset with no actuator command applied and processing it with the differential algorithm described below. The resulting frames are then analyzed to measure the noise contribution. As this method is based on real measurement, it is sensitive to all the noise sources within the measurement setup. This technique is suitable to estimate the convection and vibration noise in (e.g.) IF or any sampling when a differential acquisition is required.

To be representative, the sampling conditions of the test dataset should match as close as possible those of the final system, for instance: frame rate, system geometry, thermal configuration. Within the present scope, we proceeded by analogy and we considered for the M4U the test datasets collected in the M4DP ([6]), VLT-DSM ([5]) and LBT ([7]) optical test bench. The number of collected frames needed in a differential measurement dataset shall be large enough to allow estimating the expected noise when averaging a large number of frames.

The differential algorithm we are referring is described here. If we consider a sequence of $n$ phase maps, collected at a cadence $t$, the resulting image $S n$ is computed as:

$$
\mathrm{S}_{\mathrm{n}}=1 / \mathrm{n} \sum\left[(-1)^{\mathrm{i}} \Phi_{\mathrm{i}}+(-1)^{(\mathrm{i}-1)} \Phi_{\mathrm{i}-1}\right]
$$

where $\Phi_{\mathrm{i}}$ is the $i$-th image acquired by the interferometer within the sequence.

In the case of no command applied, the resulting image $S n$ represents the expected noise frame when averaging together a set of $n$ differential frame. The image is then analyzed to extract the wanted information: e.g. WFE, differential piston among segments, tip-tilt, slope error. 


\section{ANALYSIS STRATEGY}

The goal of the analysis is to provide a numerical error budget for the optical test tower conceived for the M4U. In particular, a general workflow applicable to each error budgeting definition can be summarized in the following steps:

- identification and list of the requirements (REQ) and constraints of the optical measurements

- identification of the verification measurement type for each requirement

- $\quad$ identification of the error sources associated with each specific measurement type

- $\quad$ identification of the analysis strategy for the specific measurement types with associated error sources

Once these first four steps are completed, the analysis can be numerically implemented ([4]) and all the individual error sources can be quantified at first order level. Therefore, they can be used as baseline input for the subsystems specifications (optics, mechanics). Each error source at this point is inserted as single voice of a budget for each given requirement ([3]).

\subsection{Identification and list of the REQ verification measurements}

We categorized the REQ per the type of verification procedure, i.e. the measurement to be performed for the REQ verification. In the following table, we indicate two types of verification measurements: differential sampling (DFS) and absolute sampling (ABS). The latter is performed both on the M4U (LAI or SAI) and on the OTT optics (without the M4U, to qualify the OTT itself). In differential samplings, we collect at a high frame rate a sequence of phase maps when applying a sequence of opposite actuator commands. The result is computed as the mean value of consecutive image pairs. In absolute samplings, we compute the average of a sequence of phase maps with the same actuator command applied.

\begin{tabular}{|c|c|c|c|}
\hline Type & Scope & REQ sample & Verif. Measurement \\
\hline \multirow[t]{2}{*}{$\begin{array}{l}\text { M4U act. } \\
\text { command } \\
\text { verification }\end{array}$} & $\begin{array}{l}\text { Command accuracy within a } \\
\text { percentage of the applied } \\
\text { command }\end{array}$ & $\begin{array}{l}\text { Accuracy of Zernike } \\
\text { command }\end{array}$ & Diff. sampling of M4U - LAI \\
\hline & $\begin{array}{l}\text { Capability of the system to } \\
\text { perform a given delta } \\
\text { movements between two } \\
\text { neighbor actuators }\end{array}$ & Inter-actuator stroke & Diff. sampling of M4U - LAI \\
\hline \multirow{3}{*}{$\begin{array}{l}\text { M4U absolute } \\
\text { shape } \\
\text { verification }\end{array}$} & $\begin{array}{l}\text { Curvature bounds on patches } \\
\text { after flattening }\end{array}$ & Flattening: Curvature & \multirow[t]{2}{*}{ Absolute sampling of M4U- LAI } \\
\hline & $\begin{array}{l}\text { Slope bounds on patches } \\
\text { after flattening }\end{array}$ & Flattening: Slope error & \\
\hline & $\begin{array}{l}\text { High spatial frequency } \\
\text { flattening result }\end{array}$ & High spatial frequency & Absolute sampling of M4U - SAI \\
\hline \multirow[t]{2}{*}{$\begin{array}{l}\text { OTT accuracy } \\
\text { verification }\end{array}$} & $\begin{array}{l}\text { OTT WFE induced to the } \\
\text { M4 measurement }\end{array}$ & WF accuracy & Absolute sampling of OTT - SAI \\
\hline & $\begin{array}{l}\text { Accuracy on segments } \\
\text { cophaseing }\end{array}$ & Co-phasing accuracy & Absolute sampling of OTT - LAI \\
\hline
\end{tabular}

Table 1 REQ verification measurements summary

\subsection{Identification of the error sources}

Starting from the optical design, we took into account the different measure procedure (DFS and ABS) and the different tower configurations (LAI, SAI and SPL) and we isolated the relevant error sources, including measurement noise and 
processing errors, to be expected during the optical test. In the following table, the error sources associated with the verification measurements are listed.

\begin{tabular}{|c|c|c|c|}
\hline Source & Error & Applicable to & Notes \\
\hline $\begin{array}{l}\text { Interferometer } \mathrm{CCD} \\
\text { readout noise }\end{array}$ & Pixel to pixel random offset & DFS, ABS & Converted into phase noise \\
\hline $\begin{array}{l}\text { Turbulence and air } \\
\text { convection }\end{array}$ & $\begin{array}{l}\text { All spatial scales changing } \\
\text { offset }\end{array}$ & DFS, ABS & \\
\hline Thermal drifts & $\begin{array}{l}\text { Alignment error (tip-tilt, power, } \\
\text { coma) }\end{array}$ & DFS, ABS & Low frequency $(<<1 \mathrm{~Hz})$ \\
\hline Vibrations & $\begin{array}{l}\text { Alignment error (tip-tilt, power, } \\
\text { coma) }\end{array}$ & DFS, ABS & $\begin{array}{l}\text { High frequency } \\
(1 \mathrm{~Hz}<\mathrm{f}<25 \mathrm{~Hz})\end{array}$ \\
\hline Re-trace error & Varying Zernike offset & DFS & $\begin{array}{l}\text { Depending on the local slope } \\
\text { (large slopes only) }\end{array}$ \\
\hline $\begin{array}{l}\text { Optics } \\
\text { manufacturing }\end{array}$ & Static offset & ABS & \\
\hline SPL accuracy & Segment differential piston error & $\mathrm{ABS}$ & \\
\hline \multirow[t]{5}{*}{ Processing errors } & $\begin{array}{l}\text { Accuracy of Zernike fitting over } \\
\text { subaperture (RM) }\end{array}$ & ABS & \\
\hline & Phase ambiguity solution & DFS, ABS & $\begin{array}{l}\text { To correct the phase maps piston } \\
\text { value }\end{array}$ \\
\hline & Tilt de-trend & DFS, ABS & To remove the vibration tilt \\
\hline & Mosaic error & ABS & $\begin{array}{l}\text { Merge the segments in a single } \\
\text { image }\end{array}$ \\
\hline & Calibration images mapping & ABS & \\
\hline
\end{tabular}

Table 2 Measurement error sources

In the Table 3, the inputs and analysis methods for each error source identified for the M4U are specified in detail and summarized.

\begin{tabular}{|l|l|l|l|}
\hline Errors source & Input from & \multicolumn{2}{|l|}{ Analysis method } \\
\cline { 2 - 4 } & & DFS & ABS \\
\hline $\begin{array}{l}\text { Interferometer CCD } \\
\text { readout noise }\end{array}$ & Lab measurements & $\begin{array}{l}\text { Processing: diff. } \\
\text { algorithm }\end{array}$ & Sample averaging \\
\hline $\begin{array}{l}\text { Turbulence and air } \\
\text { convection }\end{array}$ & $\begin{array}{l}\text { Lab measurements (DP, } \\
\text { LBT dome, DSM+ ASSIST) }\end{array}$ & $\begin{array}{l}\text { Processing: diff. } \\
\text { algorithm }\end{array}$ & $\begin{array}{l}\text { Processing: } \\
\text { averaging }\end{array}$ \\
\hline Thermal drifts & OTT FEA + optical design & $\begin{array}{l}\text { Analytical } \\
\text { computation }\end{array}$ & $\begin{array}{l}\text { Analytical } \\
\text { computation }\end{array}$ \\
\hline $\begin{array}{l}\text { Vibrations } \\
\text { Re-trace error }\end{array}$ & OTT FEA + optical design & $\begin{array}{l}\text { Analytical } \\
\text { computation }\end{array}$ & $\begin{array}{l}\text { Analytical } \\
\text { computation }\end{array}$ \\
\hline
\end{tabular}




\begin{tabular}{|l|l|l|l|}
\hline Optics manufacturing & $\begin{array}{l}\text { Literature } \\
\text { measurement }\end{array}$ & computation & $\begin{array}{l}\text { Analytical } \\
\text { computation }\end{array}$ \\
\hline SPL accuracy & Literature & n. a. & $\begin{array}{l}\text { Analytical } \\
\text { computation }\end{array}$ \\
\hline Processing errors & $\begin{array}{l}\text { Individual error sources } \\
\text { simulation }\end{array}$ & $\begin{array}{l}\text { Simulation, } \\
\text { numerical code }\end{array}$ & $\begin{array}{l}\text { Simulation, } \\
\text { numerical code }\end{array}$ \\
\hline
\end{tabular}

Table 3 Reference inputs for the measurement error analysis. DP: M4 Demonstration Prototype. LBT: Large Binocular Telescope Adaptiver secondary. DSM: VLT-UT4 adaptive secondary, tested on its optical tower ASSIST.

\subsection{Processing: a common error source}

A special role is played by the contribution of the data processing to each measurement set.

Usually the amount of error added with processing images (e.g. resampling, creation of Zernike modes) is a small percentage of the initial aberration.

Hereafter what we identified as fundamental processes that we have to run in order to properly manage the measurements:

- Alignment Zernike fitting over RF (reference flat) sub-aperture: the measurement of the Zernike alignment modes (focus and coma) in the OTT is performed using the Reference Flat mirror (RF) as a reference surface (i.e. focus and coma free) ([3], [2]). The RF is smaller than the optical surface (0.6 m vs $1.5 \mathrm{~m}$ diameter) and this is affecting the accuracy of the Zernike modes fitting, in particular in presence of a large tilt offset.

- Phase ambiguity solution: the interferometer is not sensitive to the absolute (total) piston of the test optics, so that an arbitrary $n-\lambda$ (with integer $n$ ) offset is added by the software to each phase map. When the image is separated into several islands, the offset is added to each island separately and it affects the measurement of the differential piston among the islands (which, in our case, are the segments for the M4U images). The phase ambiguity may be solved as demonstrated for the M4DP optical test ([6]).

- Tilt de-trending: the phase ambiguity solution algorithm may converge only when the tilt of the image islands is corrected. We defined a global tilt correction procedure using the partially hidden segments available in the interferometric image, allowing either the equalization of the tilt across the islands or the nulling of the global tilt. The procedure is complex and out of the scope of this section but is an important error source.

- Mosaic: the M4U will be sampled in the OTT as separated phase maps of the different segments, because of the tower optical configuration. The mosaic error is the processing error introduced when combining geometrically the segment images to produce a global M4U phase map.

- Subtraction of calibration images: the calibration images of the OTT optics will be subtracted to the absolute measurements, to remove the manufacturing offset. To perform a robust subtraction, the calibration images shall be aligned according to the current tower geometry. The alignment error will be therefore introduced in this process.

\subsection{Errors propagation to REQ and final budgeting}

We analyzed each error source in Table 3 in terms of the applicable REQs. For instance, convection noise (which is an error source common to many REQs) has been analyzed to estimate the associated slope error, curvature error, cophasing.

For those cases when the error source is a measurement noise depending on the sampling parameters (e.g. frame rate, averaging time), we set an arbitrary error value and defined the corresponding sampling parameters to achieve it in the OTT. This strategy will be discussed case by case when applied in ([3])

The same a priori approach was adopted for the budgeting of optical and mechanical subsystems. In these cases, the resulting budget was taken as a part of the subsystem specifications to be passed to the manufacturer. For the mechanics, 
for instance, we defined an internal, high level, performance budget in terms of WFE due to thermal drifts and mechanical vibrations; we started from the rough mechanical design produced for the PDR as a reference to compute the sensitivities; such budget was then converted into a set of internal specifications for the design of the OTT. We implemented a verification algorithm to check that the proposed mechanical design matches with the allocated budget. For the optics, we considered as our driving specification the WFE residual we can achieve after calibrating the OTT cavity.

In the end, all the individual results have been reported in the final error budget, each associated with its REQ.

The errors propagation from sources to REQ verification is summarized in the following chart.

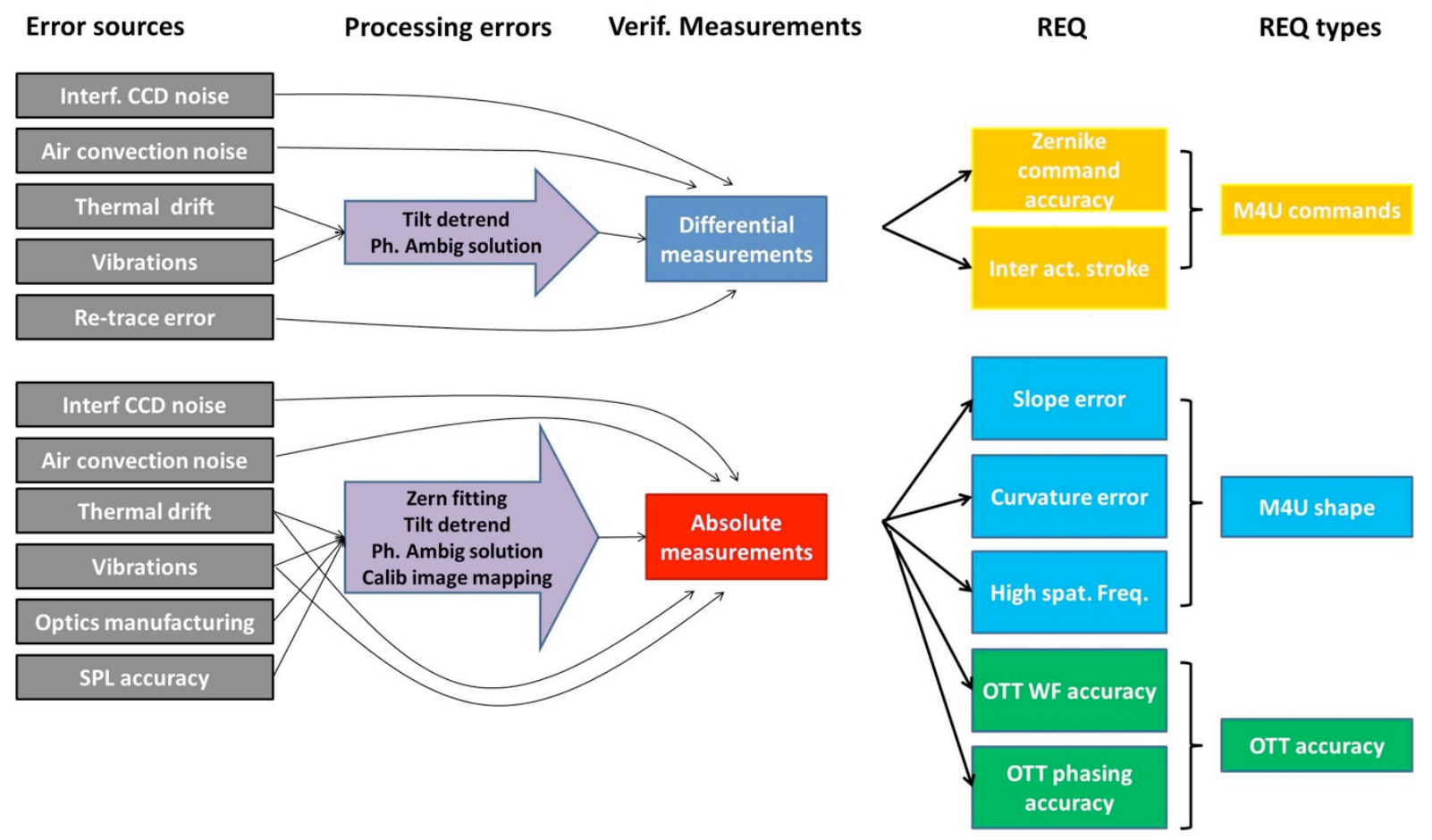

Figure 4: Errors propagation from sources to the optical REQs verification.

\section{CONCLUSIONS}

We presented in this article an analysis strategy for the budgeting of the optical measurements of the E-ELT adaptive mirror M4. The budgeting process started from the identification of the measurement types and of the error sources associated to them. The individual terms are then analyzed to evaluate their contribution and their dependence from the sampling parameters. At last, we identified the measurements needed for the verification of each of the system requirements and summed up the individual error term into the final budget.

The analysis concept was developed starting from the test cases of the LBT and VLT adaptive mirrors and tailored for the E-ELT one. Therefore, the paper may be considered as a useful guideline in the error budgeting of the optical calibrations and measurements for the large format deformable mirrors foreseen in the next generation of giant telescopes. 


\section{REFERENCES}

[1] "The E-ELT M4, on its way to become reality", Vernet E. et al., Proceeding of AO4ELT 5 conference, 2017

[2] Pariani G., Briguglio R., Xompero M., "Optical calibration of the E-ELT adaptive mirror M4: design, alignment and verification of the interferometric test tower.", Proceeding of AO4ELT 5 conference, 2017

[3] Briguglio R., Xompero M., Pariani G. "Optical calibration of the E-ELT adaptive mirror M4: testing protocol and assessment of the measurement accuracy", Proceeding of AO4ELT 5 conference, 2017

[4] Briguglio, R., Pariani, G., Xompero, M. \&al " 8 s, a numerical simulator of the challenging optical calibration of the E-ELT adaptive mirror M4"; Society of Photo-Optical Instrumentation Engineers SPIE) Conference Series, 9909. 2016.

[5] Briguglio, R., Riccardi, A., Xompero, M.: "Optical Test Report (VLT-TRE-OAA-112504054_Optical_Test_Report)", October 2013.

[6] Briguglio, R., Xompero, M., Riccardi, A., Pariani, G. et al: "Optical calibration of the M4 prototype toward the final unit", Proceedings of the 4th AO4ELT conference, November 2015.

[7] Riccardi, A., Xompero, M., Briguglio, R., et al: "The adaptive secondary mirror for the Large Binocular Telescope: optical acceptance test and preliminary on-sky" 